\title{
Dynamics of the Endoplasmic Reticulum and Other Membranous Organelles in Growth Cones of Cultured Neurons
}

\author{
Michael E. Dailey and Paul C. Bridgman \\ Department of Anatomy and Neurobiology, Washington University School of Medicine, St. Louis, Missouri 63110
}

The fluorescent lipophilic dye 3,3'-dihexyloxacarbocyanine iodide $\left[\mathrm{DiOC}_{6}(3)\right]$ was used to examine the distribution of membrane-bound organelles in growth cones of cultured rat sympathetic neurons. Within chemically fixed growth cones, intense $\mathrm{DiOC}_{6}(3)$ fluorescence was localized predominately to the base or central region of growth cones. However, in most growth cones several thin $\mathrm{DiOC}_{6}(3)$-fluorescent processes radiated from the base into the periphery, and double fluorescence imaging of single growth cones indicated that these processes were highly colocalized $(\sim 79 \%)$ with microtubules.

The distribution of $\mathrm{DiOC}_{6}(3)$ fluorescence in living growth cones was examined using low light-level fluorescence video microscopy. We observed thin fluorescent processes within the periphery of growth cones to undergo length excursions (extension/retraction) and to change orientation (move laterally). During growth cone advance, processes became progressively thicker and were gradually engulfed by the advancing fluorescent mass. When growth cones were viewed with video-enhanced differential interference contrast microscopy, the position of the fluorescent processes correlated with thickened extensions of central-type cytoplasm through which vesiclelike organelle transport often occurred.

These observations indicate several features concerning the organization and movement of membranous organelles (MOs) in growth cones: (1) MOs are highly compartmentalized, the majority being localized to the growth cone base; (2) MOs advance into the periphery along distinct pathways probably associated with microtubules; (3) one or more thin continuous MOs, which most likely represent a thin tubular component of the endoplasmic reticulum, generally precedes advance of vesiclelike MOs along individual transport pathways; and (4) transport pathways with their associated MOs are spatially and temporally dynamic.

There is strong evidence to indicate that the endoplasmic reticulum (ER) is a major regulator of cell calcium (Somlyo, 1984; Carafoli, 1987). Within neurons the ER, including the axoplasmic reticulum, is able to sequester calcium (Henkart et al., 1978; Wal7, 1982; Unger et al., 1984; Burton and Laveri, 1985),

\footnotetext{
Received June 24, 1988; revised Sept. 29, 1988; accepted Nov. 2, 1988.

We wish to thank Dr. Mark Terasaki for helpful discussions and generously providing details of protocols, and Dr. Naomi Klietman for helpful comments on the malluscripl. This work was supported by NIH Grants GM08151 and NS26150.

Correspondence should be addressed to Dr. Paul C. Bridgman, Washington University School of Medicine, Department of Anatomy/Neurobiology, Box 8108, 660 S. Euclid Avc., St. Louis, MO 63110.

Copyright $(1989$ Society for Neuroscience $0270-6474 / 89 / 061897-13 \$ 02.00 / 0$
}

and recent evidence suggests that the ER within neurons and neuronal processes can release stored calcium in response to external signals (Neering and McBurney, 1984; Payne and Fein, 1987; Lipscombe et al., 1988a, b).

It is widely believed that calcium may be an important intracellular regulator of neurite elongation and growth cone motility. Measurements of calcium using a fluorescent indicator have revealed a correlation between the level of calcium in growth cones and neurite extension (Connor, 1986; Cohan et al., 1987), and the effect of factors such as serotonin and action potentials, which inhibit growth cone motility (Cohan and $\mathrm{Ka}$ ter, 1986), is apparently mediated by intracellular calcium (Cohan et al., 1987). These studies suggest a relationship between calcium levels and growth state of growth cones (Cohan et al., 1987).

The experiments of Gundersen and Barrett (1980) demonstrated that growth cones from chick sensory neurons in culture turn toward reagents known to increase intracellular calcium levels. Interestingly, the turning response was elicited by conditions that permit calcium influx as well as conditions that stimulate calcium release from internal stores. In addition, the turning response of growth cones toward a source of nerve growth factor persisted in the virtual absence of extracellular calcium, although the combination of low $\left(10^{-9} \mathrm{M}\right)$ calcium and dantrolene (an inhibitor of calcium release from intracellular stores) in the perfusion bath abolished the turning response (Gundersen and Barrett, 1980). Thus, intracellular calcium may be important for the ability of growth cones to sense and respond to putative directional cues.

Recent observations of growth cones in culture using videoenhanced light microscopy indicate the presence of distinct domains in relation to organelle movements (Goldberg and Burmeister, 1986; Forscher et al., 1987), and these domains have been correlated with regional differences in cytoplasmic organization (Bridgman et al., 1986; Forscher et al., 1987). Advance of growth cones involves infiltration of organelles into previously organelle-free cytoplasmic regions, and this is apparently associated with a process of cytoplasmic "maturation" reflected in locally reduced motility and increased stability (Goldberg and Burmeister, 1986; Aletta and Greene, 1988). Studies of nonneuronal cells in culture have revealed a close spatial relationship of microtubules (MT) and tubules of ER within thinly spread regions of cells (for example, Buckley and Porter, 1975; Terasaki et al., 1986), and it was recently proposed that the ER extends into new cytoplasmic regions in association with $\mathrm{mi}$ crotubules to stabilize the cytoplasmic structure, presumably by regulating local calcium levels (Terasaki et al., 1986).

These observations have led us to study the distribution and morphology of the endoplasmic reticulum within growth cones 
to help establish a morphological framcwork within which the function of the growth cone ER may be considered. As growth cones are highly dynamic regions of neurons, it would be advantageous to examine ER structure in relation to the activity of living growth cones observed in the light microscope. Recently, 3,3'-dihexyloxacarbocyanine iodide $\left[\mathrm{DiOC}_{6}(3)\right]$, a fluorescent lipophilic dye, has been employed to study with the light microscope the morphology of the ER in fixed and living cultured cells (Terasaki et al., 1984, 1986; Lee and Chen, 1988). We have extended the use of $\operatorname{DiOC}_{6}(3)$ to study membranous organelles, including the ER, in growth cones of cultured neurons. We report that $\mathrm{DiOC}_{6}(3)$ stains the entire base of growth cones as well as fingerlike processes that extend from the base toward the margin. The fingerlike processes are highly colocalized with microtubules, and in living growth cones the processes extend, retract, and move laterally over time. During growth cone advance the processes gradually thicken until they arc cngulfed by the fluorescent mass at the base of the growth cone. We suggest that the fingerlike processes of $\mathrm{DiOC}_{6}(3)$ fluorescence observed in the light microscope include fine tubules of endoplasmic reticulum, often in association with microtubules, and that these tubules of ER are highly dynamic within the periphery of growth cones. A portion of these results has been presented previously (Dailey and Bridgman, 1987).

\section{Materials and Methods}

Tissue culture methodology. Explants of embryonic day 21 rat superior cervical ganglia (SCG) were prepared and cultured on laminin-coated glass coverslips as described elsewhere (Bridgman and Dailey, 1989). Growth cones were examined on the second day in culture (12-24 hr after explantation).

$D i O C_{6}(3)$ staining of cultures. Protocols for staining fixed and living cultures were essentially the same as reported by Terasaki et al. (1984). To chemically fix cultures, $1 \mathrm{ml}$ of warm $\left(35^{\circ} \mathrm{C}\right)$ fixative $(0.25 \%$ glutaraldehyde in $0.1 \mathrm{M}$ cacodylate buffer containing $5 \mathrm{~mm}$ calcium chloride) was added dropwise to cultures immediately following removal from the incubator. After 2-5 min, cultures were rinsed several times with fresh buffer by adding and removing buffer from the culture dish with a pipette.

A stock solution of dye was made by adding $0.5 \mathrm{mg} \mathrm{DiOC}$ (3) (Molecular Probes, Junction City, OR) to $1 \mathrm{ml}$ ethanol. The working solution was made on the day of use by diluting the stock in buffer to a final concentration of $2.5 \mu \mathrm{g} / \mathrm{ml}$. To stain fixed cultures, buffer was removed from the cultures and $2 \mathrm{ml}$ of $\mathrm{DiOC}_{6}(3)$-containing buffer was added to each dish. After $20 \mathrm{sec}$, the dye was removed, and the cultures were rinsed with fresh buffer.

To stain living cultures, $100 \mu \mathrm{l}$ of a solution of $\mathrm{DiOC}_{6}(3)(3 \mu \mathrm{l}$ stock in $1 \mathrm{ml}$ culture medium, warmed to $35^{\circ} \mathrm{C}$ ) was added to each culture dish (final concentration, $0.3 \mu \mathrm{g} / \mathrm{ml}$ ). The cultures wcre returned to the incubator for $10 \mathrm{~min}$ and then removed and mounted with fresh dyefree culture medium $\left(35^{\circ} \mathrm{C}\right)$ for observation.

Analysis of neurite outgrowth. Six hours after explantation, some cultures were stained with $\operatorname{DiOC}_{6}(3)(0.3 \mu \mathrm{g} / \mathrm{ml})$ for $10 \mathrm{~min}$, then rinsed with fresh culture medium and returned to normal culture conditions. Control cultures from the same batch were not exposed to dye but otherwise treated identically. Fourteen hours later, experimental and control cultures were fixed, mounted, and photographed with a $35 \mathrm{~mm}$ camera (Contax) attached to an inverted microscope (Zeiss IM35) equipped with a $2.5 \times$ objective. Photographs were taken of all explants and neurite fields on the coverslips, and measurements of the maximal neurite outgrowth for each explant were taken directly from the photographic negatives. (The maximal neurite outgrowth of an explant was defined as the greatest radial distance from the edge of the cxplant to the perimeter of the neurite field.)

Fluorescence microscopy. Fixed cultures were prepared for microscopic observation by removing the coverslip with explants from the dish and mounting with buffer on a glass microscope slide. Two thin strips of no. 0 coverglass were used as spacers between the microscope slide and coverslip. Exccss buffer was removed and the chamber was sealed with hot wax. Culture chambers were mounted on an inverted microscope equipped with a $63 \times$ Planapo oil-immersion objective (Zeiss). $\mathrm{DiOC}_{6}(3)$ fluorescence was visualized using a fluorescein filter system and epi-illumination. Explants with substantial neurite outgrowth were located, and the perimeter of the neurite fields were searched for growth cones. Growth cones were photographed using Kodak Tri-X Pan or T-Max film.

Living, $\mathrm{DiOC}_{6}(3)$-stained cultures were mounted in a closed chamber as described above, except that a no. 0 glass coverslip was used in place of the microscope slide. The chamber was secured to a metal holder with tape, and the entire assembly was placed on the heated $\left(35^{\circ} \mathrm{C}\right)$ stage of an inverted microscope. The chamber temperature was continuously monitored by a miniature thermocouple attached to the coverslip with thermoconductivc paste (Omega Engineering, Stamford, CT). Initially, growth cones were viewed with a silicon-intensified target (SIT) camera (General Electric) and video-imaging system, including a real-time image processor (Hughes Aircraft Co., model 794) and U-matic videocassette recorder (Sony, model VO-5800). The light source was a 100 W halogen lamp (Zeiss) operated at $4.5-6 \mathrm{~V}$ and controlled' with a manual shutter. Frame averaging was necessary to reduce noise in the video image obtained under the low-intensity light. Fluorescence images of growth cones were viewed on the video monitor for focusing, immediately captured with the image processor, and recorded on video tape. Thus, growth cones were illuminated for only a brief duration $(<10 \mathrm{sec})$ to focus and capture each image. Images of single growth cones were captured and recorded at approximately 1, 2, or $4 \mathrm{~min}$ intervals. Later, stored images were retrieved and photographed directly from the vidco monitor.

During the course of these experiments we acquired an image intensifier (Videoscope International, Washington, D.C.) with manually controlled gain, which we attached to a Newvicon camera (Ikegami, Tokyo). This configuration, coupled with a $20 \times$ projection eyepiece, permitted us to obtain higher-resolution images at lower illumination intensities than the imaging configuration described above. Typically, the illuminating voltage of the $100 \mathrm{~W}$ halogen lamp was reduced to between 2$4 \mathrm{~V}$, resulting in an increase in the viability of observed living specimen (see Results). Acquisition of an optical disk recorder (Panasonic, model TQ-2028F) permitted us to record high-quality images rapidly and thus further reduce the exposure time of the specimen to light.

Sequential DiOC $C_{6}(3)$ and anti-tubulin fluorescence technique. The fixation and double-fluorescence procedure of Terasaki et al. (1986) was used to image $\mathrm{DiOC}_{6}(3)$ fluorescence and microtubules in single growth cones. Briefly, cultures were fixed, stained with $\operatorname{DiOC}_{6}(3)$, and photographed as described above. Subsequently, cultures were permeabilized $(0.2 \%$ Triton X-100, 2 min) and processed for indirect immunofluorescence by the method of McBeath and Fujiwara (1984) to visualize microtubules (primary: rabbit anti-sea urchin alpha-tubulin (Polysciences), 1:40, $1 \mathrm{hr}$; secondary: rhodamine-conjugated anti-rabbit IgG (Cappel), 1:400, $45 \mathrm{~min}$ ). Cultures were remounted, and growth cones were photographed using a rhodamine filter system. To facilitate finding the same growth cone, photographs were taken of many growth cones at the perimeter of neurite fields.

Analysis of $\mathrm{DiOC}_{0}(3)$ and microtubule colocalization. Photographic images of $\mathrm{DiOC}_{6}(3)$ and anti-tubulin fluorescence were printed at a final magnification of $2620 \times$. A clear acetate sheet was placed over each image, and the pattern of $\mathrm{DiOC}_{6}(3)$ or anti-tubulin fluorescence was traced within a silhouette of the growth cone. The silhouette was traced from the $\mathrm{DiOC}_{6}(3)$ image. Quantitative analyses of the $\mathrm{DiOC}_{6}(3)$ and microtubule colocalization excluded much of the growth cone base (see Results for rationale). For each growth cone, the total length of microtubule segments $(M T)$ was calculated from direct measurements of the traced anti-tubulin image. Then, the traced image of $\mathrm{DiOC}_{6}(3)$ fluorescence was superimposed upon the traced anti-tubulin image, and the total length of microtubules not covered by the $\operatorname{DiOC}_{6}(3)$ image $\left(M T_{\text {uc }}\right)$ was determined by direct measurements. The percentage of microtubule length colocalized with $\mathrm{DiOC}_{6}(3)$ fluorescence was calculated using the formula: $100 \times\left(M T_{1}-M T_{\mathrm{uc}}\right) / M T_{\mathrm{r}}$.

Video-enhanced differential interference contrast microscopy. Neuronal cultures were established as described above and growth cones were observed in real time (3-30 min) or after fixation with video-enhanced contrast-differential interference contrast (VEC-DIC) microscopy using the inverted microscope $(63 \times$ objective) and video setup described above, except for the following modifications. A $100 \mathrm{~W}$ mercury arc lamp adjusted for critical illumination was the light source, and the specimen was viewed with a Newvicon camera (Ikegami) coupled with 
a $25 \times$ eyepiece. To reduce the heat load on the specimen, heat reflection and heat adsorption filters were placed in series in the path of illumination. The light was made monochromatic $(546 \mathrm{~nm})$ using an interference filter. Real-time background subtraction was used to obtain a mottle-free image.

To rapidly fix living specimen after observation, the coverslips were mounted in a simple chamber with small openings (covered by glass slivers) on either end for perfusion. At the end of the observation period, the condenser was racked away from the chamber and the glass slivers were moved aside to expose the chamber medium. Fixative was added dropwise to one side of the chamber and medium was withdrawn simultaneously on the other side. Thus, fixative could be added to the chamber in less than 15 sec after the last VEC-DIC image. A final VECDIC image of the specimen was obtained to confirm successful fixation Some observed cultures were stained with $\mathrm{DiOC}_{6}(3)$ after fixation by perfusing the stain in the same manner as the fixative. The microscope was prepared for fluorescence as described above, except that a $50 \mathrm{~W}$ mercury lamp was used, and $\mathrm{DiOC}_{6}(3)$ image was captured with a SIT camera and recorded on video tape.

\section{Results}

\section{General features of cultures and $\mathrm{DiOC}_{6}(3)$ staining}

Explants of embryonic rat superior cervical ganglia exhibited vigorous neurite outgrowth when grown on a laminin-containing substratum. Neurites extended radially from the explant about $1 \mathrm{~mm}$ within $24 \mathrm{hr}$, and numcrous growth cones were found at the perimeter of the neurite fields. When conditions of fixation were optimal, that is, when cultures were fixed quickly after removal from the incubator with warm fixative, the cultures contained rather large (10-40 $\mu \mathrm{m})$ growth cones with broad lamellipodia.

Neurite fields of cultures were often infiltrated by non-neuronal cells that migrated onto the substratum from the explant. The presence of non-neuronal cells within the neuronal cultures provided an internal standard for the fixation and $\mathrm{DiOC}_{6}(3)$ staining procedures. Staining of fixed cultures with $\mathrm{DiOC}_{6}(3)$ revealed an elaborate reticular pattern of fluorescence within the thin, peripheral regions of non-neuronal cells (Fig. 1A). In some non-neuronal cells, free-ending processes extended from the fluorescent network toward the cell margin. In addition, a bright punctate staining was apparent overlying the reticular network, though it was absent from the most peripheral regions of cells. The punctate staining presumably represents staining of mitochondria and vesicular organelles which are prevalent in perinuclear cell regions. Staining of the plasma membrane was faint. These observations are consistent with previous reports of $\mathrm{DiOC}_{6}(3)$-stained non-neuronal cells in which the dye has been demonstrated to stain the endoplasmic reticulum, as well as mitochondria, vesicular organelles, and the plasma membrane (Johnson et al., 1981; Terasaki et al., 1984).

\section{$\mathrm{DiOC}_{6}(3)$ fluorescence of fixed growth cones}

Neurites of $\mathrm{DiOC}_{6}(3)$-stained cultures were fluorescent along their entire length, although the intensity varied. In particular, there appeared a number of intensely fluorescent, elongate (up to $8 \mu \mathrm{m}$ long) figures that were oriented along the neurite axis. The shape and size of the figures are consistent with that of mitochondria (Johnson et al., 1981). $\mathrm{DiOC}_{6}(3)$ fluorescence invariably extended from the distal neurite into the base of growth cones.

In many growth cones, no segments or spots of intense fluorescence were found that were not contiguous with the fluorescence at the growth cone base. However, in a number of fixed growth cones, isolated fluorescent spots of variable size were found in the periphery. The number of spots per growth cone ranged from 1 or 2 to several and was apparently correlated with the rate of fixation and the concentration of glutaraldehyde in the fixative. In growth cones that were fixed, stained with $\mathrm{DiOC}_{6}(3)$, and observed with fluorescence and VEC-DIC microscopy, many of the isolated fluorescent spots corresponded to localized areas of membrane distortion such as blebbing, which has been determined to be artifactually induced by chemical fixation (Rees and Reese, 1981). In addition, regions of growth cones that were ruffling or overlapping (either with an adjacent region of the same growth cone or with a portion of another growth cone) were intensely fluorescent. Thus, we focused our analyses on growth cones that appeared to suffer minimally from fixation-induced artifact and that were not physically contacting other growth cones.

The pattern of $\operatorname{DiOC}_{6}(3)$ fluorescence within growth cones was characterized by regional variation (Fig. $1, B-D$ ). Invariably there was a rather broad region of intense fluorescence usually located at the base of the growth cone [hereafter referred to as the $\mathrm{DiOC}_{6}(3)$-fluorescent mass]. In some cases the fluorescent mass was located in the center or along one side of the growth cone, although in every instance the fluorescence was continuous between the mass and the distal neurite. As in neurites, mitochondria were often distinguishable as especially bright elongate structures within the fluorescent mass.

In contrast to the predominantly reticular pattern of $\mathrm{DiOC}_{6}(3)$ fluorescence within thinly spread regions of non-neuronal cells (Fig. 1A), most fluorescent processes within the periphery of growth cones were free-ending (Fig. 1, $B-D$ ). Distinct fingerlike processes radiated from the fluorescent mass toward the growth cone margin. These processes did not form a typical reticular network, which is characterized by 3 -way branch points (Terasaki et al., 1986). Instead, processes were often spatially isolated from one another. Processes that did interact spatially with one another either coursed roughly parallel with or appeared to cross one another (characterized by a 4 -way branch point). The number of processes within a single growth cone ranged from 0 to 13, though most cones contained at least 1 process.

Within individual growth cones, the fluorescent processes were of variablc morphology and distribution. Processes often radiated from the fluorescent mass into all parts of the growth cone periphery (Fig. 1B), though in some growth cones a large portion of the periphery was devoid of processes. Processes within a single growth cone often had divergent orientations, some being aligned with filopodia. Some processes were straight (Fig. 1C), and others were sinuous or gently bending. Some processes appeared as a single linear element, while others were comprised of 2 or 3 parallel linear elements. Distinct punctate spots of fluorescence were often visible within a process, and these spots tended to be associated with the thicker processes. The length of individual processes was variable, some being up to $20 \mu \mathrm{m}$ long. Despite the heterogeneity in appearance, all processes exhibited 2 invariant attributes: $\mathrm{DiOC}_{6}(3)$ fluorescence was continuous along the length of the process and contiguous with the fluorescent mass at the growth cone base.

\section{Colocalization of microtubules and $\mathrm{DiOC}_{6}(3)$ fluorescence}

To examine the possibility that the $\mathrm{DiOC}_{6}(3)$-fluorescent processes in the periphery of growth cones are associated with microtubules, the distributions of $\mathrm{DiOC}_{6}(3)$ fluorescence and microtubules in single growth cones were determined. Cultures were fixed and consecutively processed for $\mathrm{DiOC}_{6}(3)$ fluores- 

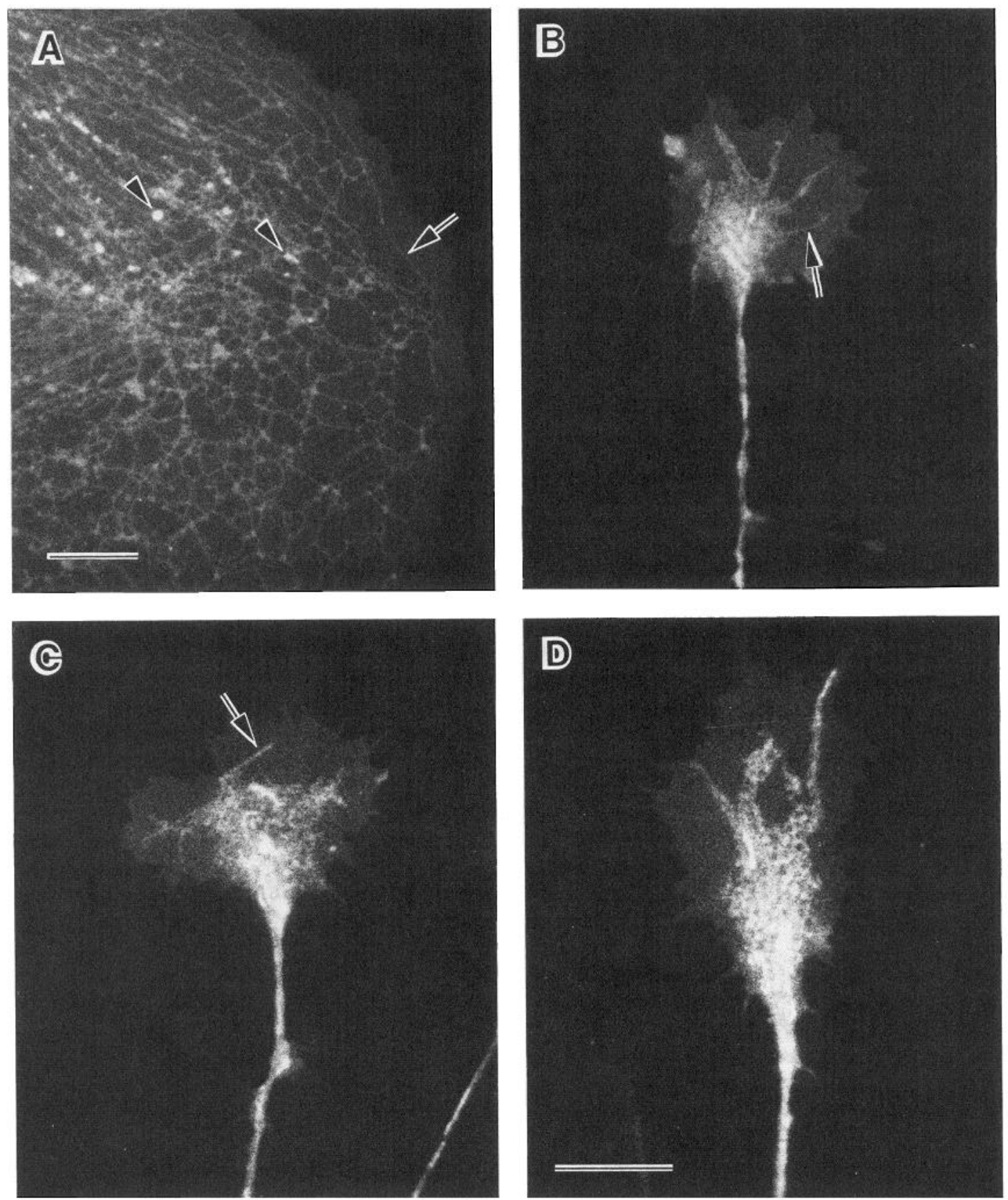

Figure 1. Fluorescent micrographs of fixed and $\mathrm{DiOC}_{6}(3)$-stained non-neuronal cell $(A)$ and neuronal growth cones $(B-D) . A$, Peripheral region of a non-neuronal cell. Note intense staining of ER (arrow), as well as other discrete organelles (arrowheads). Staining of the plasmalemma is faint. Scale bar, $10 \mu \mathrm{m} . B-D, \mathrm{DiOC}_{6}(3)$ stains the base of growth cones as well as fingerlike processes (arrows) that extend into the periphery. No reticular structure is evident. Scale bar, $10 \mu \mathrm{m}$.

cence and anti-tubulin immunofluorescence. The region of greatest concentration of microtubules within growth cones was invariably the region of greatest $\mathrm{DiOC}_{6}(3)$ fluorescence, that is, the $\mathrm{DiOC}_{6}(3)$ mass (Fig. 2). Several microtubules extended through the base and terminated within the peripheral regions of growth cones, and the distal-most segments of microtubules often appeared to correspond to $\mathrm{DiOC}_{6}(3)$-fluorescent processes (compare Fig. 2, $A, C, E$ with $B, D, F$, respectively).

Colocalization of $\mathrm{DiOC}_{6}(3)$ fluorescence and microtubules was determined by superimposing images traced from fluorescence micrographs of single growth cones. When the traced image of
$\mathrm{DiOC}_{6}(3)$-fluorescence was superimposed upon the anti-tubulin image, the images overlapped significantly. In some cases, a thin $\mathrm{DiOC}_{6}(3)$-fluorescent process appeared to duplicate precisely the sinuous course of an individual microtubule within the growth cone periphery (Fig. 2, E, F). However, not all microtubules had corresponding $\mathrm{DiOC}_{6}(3)$ fluorescence along their entire length. For example, some microtubules extended beyond processes of $\mathrm{DiOC}_{6}(3)$ fluorescence (Fig. $2 D$, arrowheads). Less frequently, the $\mathrm{DiOC}_{6}(3)$ process extended beyond the corresponding microtubule (Fig. $2 C$, arrowhead).

Determination of colocalization at the base of the growth cone 

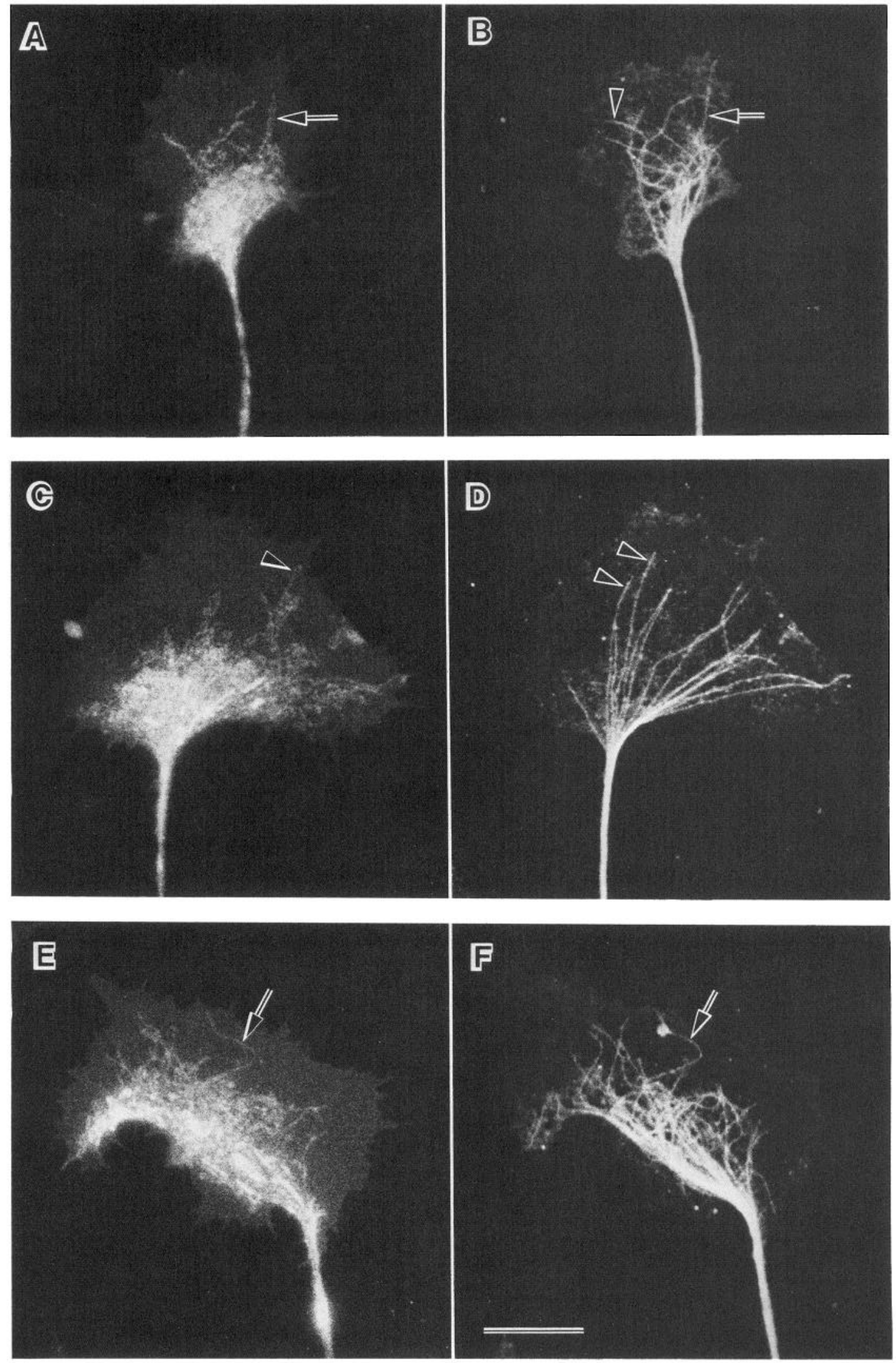

Figure 2. Fluorescent images of 3 fixed growth cones consecutively stained with $\operatorname{DiOC}_{6}(3)(A, C, E)$ and anti-tubulin anti-bodies $(B, D, F)$. $\mathrm{DiOC}_{6}(3)$-fluorescent processes often colocalize with distal segments of microtubules $(A, B$, arrows), although some microtubule segments are not associated with $\mathrm{DiOC}_{6}(3)$ fluorescence $\left(B, D\right.$, arrowheads). Less frequently, $\mathrm{DiOC}_{6}(3)$ extends beyond microtubules $(C$, arrowhead $)$. Note the precise codistribution of $\operatorname{DiOC}_{6}(3)$ fluorescence with a bending microtubule $(E, F)$. Scale bar, $10 \mu \mathrm{m}$. 
was complicated by practical considerations because the $\mathrm{Di}$ $\mathrm{OC}_{6}(3)$-fluorescent mass was located within the central cytoplasmic domain of growth cones. The central domain is a relatively thick region of the growth cone, so that overlapping fluorescence patterns may not represent strict spatial colocalization. Hence, the region of growth cones occupied by the $\mathrm{DiOC}_{6}(3)$ mass was excluded from colocalization analyses.

Quantitative analysis of colocalization of $\mathrm{DiOC}_{6}(3)$ fluorescence and microtubules in the periphery of growth cones was performed using measurements taken directly from traced images. For each growth cone, the length of microtubules with corresponding $\operatorname{DiOC}_{6}(3)$ fluorescence was expressed as a percentage of the total length of microtubule segments within the growth cone periphery. The average for all growth cones measured was $79 \pm 14 \%$ (mean $\pm \mathrm{SD}$; range, $55-100 \% ; n=21$ ) correspondence of $\mathrm{DiOC}_{6}(3)$ fluorescence with microtubules. There was no correlation between the relative extent of colocalization and the number of microtubules that projected into the growth cone periphery (data not shown). Quantitative anal$y$ sis to determine the area of $\operatorname{DiOC}_{6}(3)$ fluorescence that did not correspond to microtubules was not performed, although visual inspection suggested that this area was small (Fig. 2).

\section{$\mathrm{DiOC}_{6}(3)$ staining and imaging of living cultures: effect on neurite elongation and growth cone morphology}

To examine the effect of $\mathrm{DiOC}_{6}(3)$ on neurite elongation and growth cone morphology, living neuronal cultures were stained with a reduced concentration $(0.3 \mu \mathrm{g} / \mathrm{ml})$ of dye. The dye is reportedly toxic to mitochondria at the higher concentration used to stain fixed specimen (Terasaki et al., 1984). However, at the reduced concentration, the dye had no deleterious effects on the rate of neurite elongation since neurite outgrowth after $20 \mathrm{hr}$ in culture was not significantly different for stained $(0.87$ $\pm 0.08 \mathrm{~mm}, n=16)$ or unstained $(0.81 \pm 0.07 \mathrm{~mm}, n=16)$ cultures. Furthermore, growth cones in stained cultures appeared as healthy as those in control cultures; that is, the average number of filopodia and average area of growth cones were similar for stained and unstained cultures (data not shown). Thus, imaging of $\operatorname{DiOC}_{6}(3)$ fluorescence in living, motile growth cones was undertaken.

$\mathrm{DiOC}_{6}(3)$-stained living growth cones were initially viewed with a SIT camera (see Materials and Methods). Continuous illumination of the specimen caused growth cone retraction or collapse within $5 \mathrm{~min}$, but intermittent illumination $(\sim 10 \mathrm{sec}$ of illumination at $2 \mathrm{~min}$ intervals) extended the observation period to 10-15 min before deleterious effects became apparent. (We found that, within our culture system, a reliable indication of a growth cone with deteriorating health was retraction of all fluorescent processes, an irregularly-shaped margin, and very long spindly filopodia. Growth cones with this morphology invariably failed to advance or retracted.) Use of the intensifiercoupled Newvicon camera enabled the illuminating voltage to be lowered significantly with the result that motile growth cones could be observed intermittently for at least $1.5 \mathrm{hr}$ with no apparent deleterious effect.

While reduced intensity and duration of illumination certainly contributed to the viability of the observed specimen, it should be noted that the amount of dye incorporated into the specimen and available for excitation by the illuminating light is probably an additional factor. We observed that specimens stained for greater lengths of time (10-30 $\mathrm{min})$ or with higher concentrations of dye $(0.3-0.6 \mu \mathrm{g} / \mathrm{ml})$ appeared brighter and were appar- ently more susceptible to deleterious effects at a given illumination intensity, but that further reducing the illumination intensity during observation allowed the specimen to recover normal morphology and activity. In this regard, the manual gain on the intensifier and/or the illuminating voltage were adjusted with each observation to maintain image quality without compromising specimen health.

\section{$\mathrm{DiOC}_{6}(3)$ fluorescence of living growth cones}

As in fixed growth cones, the pattern of fluorescence in living growth cones was characterized by a mass of intense fluorescence at the growth cone base and several processes of fluorescence projecting into the periphery. The plasmalemma was faintly visible, providing a means by which the overall morphology of observed growth cones could be monitored.

Fluorescent processes within the periphery of living growth cones were observed to undergo length excursions and changes in orientation over time. Specifically, individual processes exhibited extension, retraction, bending, and lateral movements. Extension of a process into the growth cone periphery often proceeded over a period of minutes (Fig. 3). Many processes appeared to extend all the way to the growth cone margin (Fig. 4 , \#2), even to the point of entering the base of a filopodium (Fig. 5, \#6), although some clearly became quiescent or retracted before reaching the margin. Some processes were stable for sevcral minutcs following cxtension (Fig. 3, \#2), while others retracted almost immediately (Fig. 4, \#1). Retraction often resulted in complete resorption of a process, though on rare occasions we observed a process to retract only slightly before re-extending (Fig. 5, \#1). Thus, we did not consistently observe individual $\mathrm{DiOC}_{6}(3)$-fluorescent processes to undergo rounds of extension and retraction.

Changes in process orientation were accomplished by 3 methods: bending, lateral movement, and partial retraction/re-extension. During or following extension, many processes changed orientation by bending or moving laterally within the periphery (Fig. 3, \#2; Fig. 5, \#2). This sometimes resulted in crossing of processes (Fig. 3), although a crossing pattern could also result from de novo extension of a process across existing processes. Processes within the growth cone periphery could become reoriented by partially retracting and re-extending in a different direction (Fig. 5, \#1). While many processes were oriented perpendicular to the growth cone margin, portions of processes, or entire processes, which were oriented parallel to the margin often appeared to be swept backward into the growth cone base (Fig. 5 , \#1,2,4,6). It should be noted that reorientation often resulted in the alignment of a process with a prominent filopodium (Fig. 5 , \#2).

In some instances adjacent fluorescent processes exhibited synchronous behavior; that is, they extended or retracted together (Fig. 3). Even processes with disparate orientations were observed to extend synchronously (Fig. 5, \#3, 4). However, we also observed instances in which a process extended while an adjacent process retracted. Apparently, processes undergo extension and retraction independently.

We did not find a consistent correlation between the extension or retraction of $\mathrm{DiOC}_{6}(3)$-fluorescent processes and local growth cone protrusion. Processes were present in both advancing and retracting regions of growth cones; in fact, we observed processes to actively extend into advancing, stable, and retracting areas. Furthermore, there was no consistent temporal sequence when both plasma membrane and local $\mathrm{DiOC}_{6}(3)$-fluorescent pro- 

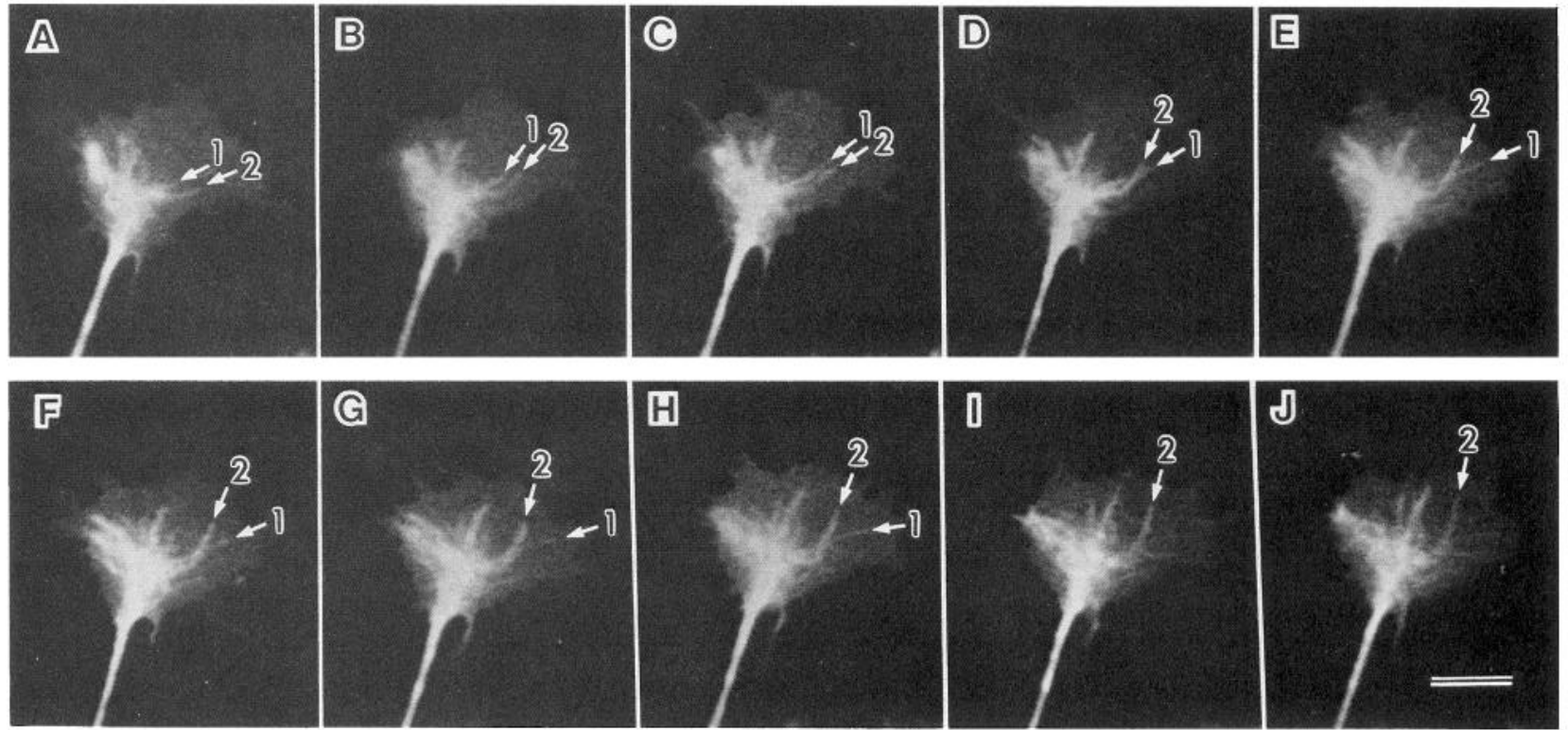

Figure 3. Time-lapse sequence of $\mathrm{DiOC}_{6}(3)$-stained living growth cone showing extension and crossing of fluorescent processes. Intervals between images are $2 \mathrm{~min}(A, B)$ or $1 \mathrm{~min}(B-J)$. Growth cone remained stationary during the $10 \mathrm{~min}$ recording session. Fingerlike processes $(1,2)$ extended simultaneously into the growth cone periphery, and process 2 subsequently moved laterally across process $l$ (cf. $C$ and $D$ ). Process 2 appeared to thicken somewhat, although 1 remained thin $(H)$. Scale bar, $10 \mu \mathrm{m}$.

cesses advanced. For example, we observed local process extension simultaneous with active protrusion of a growth cone region, but we also observed processes to extend up to the growth cone margin just prior to local membrane protrusion (Fig. 4,
\#2), as well as process extension into a region sometime after protrusion of the margin (Fig. 5, \#6). Also, plasma membrane protrusion sometimes occurred in regions of growth cones devoid of $\mathrm{DiOC}_{6}(3)$-fluorescent processes.
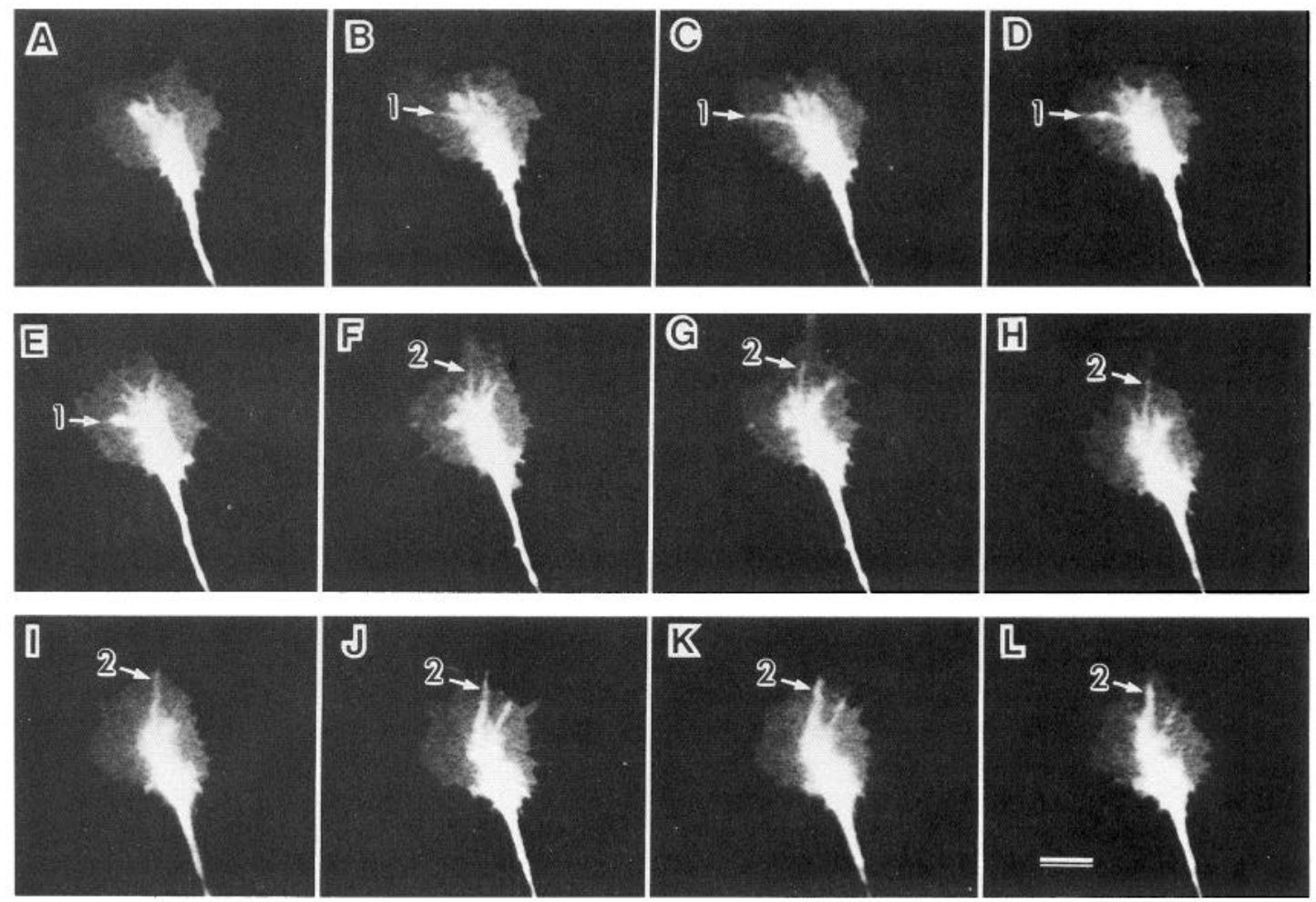

Figure 4. Time-lapse sequence of $\mathrm{DiOC}_{6}(3)$-stained living growth cone showing extension, retraction, and thickening of fluorescent processes. Process 1 extended toward margin and immediately retracted. Process 2 extended as a thin process into the base of a filopodium and became thicker; it was initially distinct from the fluorescent mass $(G, H)$ until the latter began to advance into the processes $(I-L)$. Total sequence time was $11 \mathrm{~min}$ at $1 \mathrm{~min}$ intervals. Scale bar, $10 \mu \mathrm{m}$. 

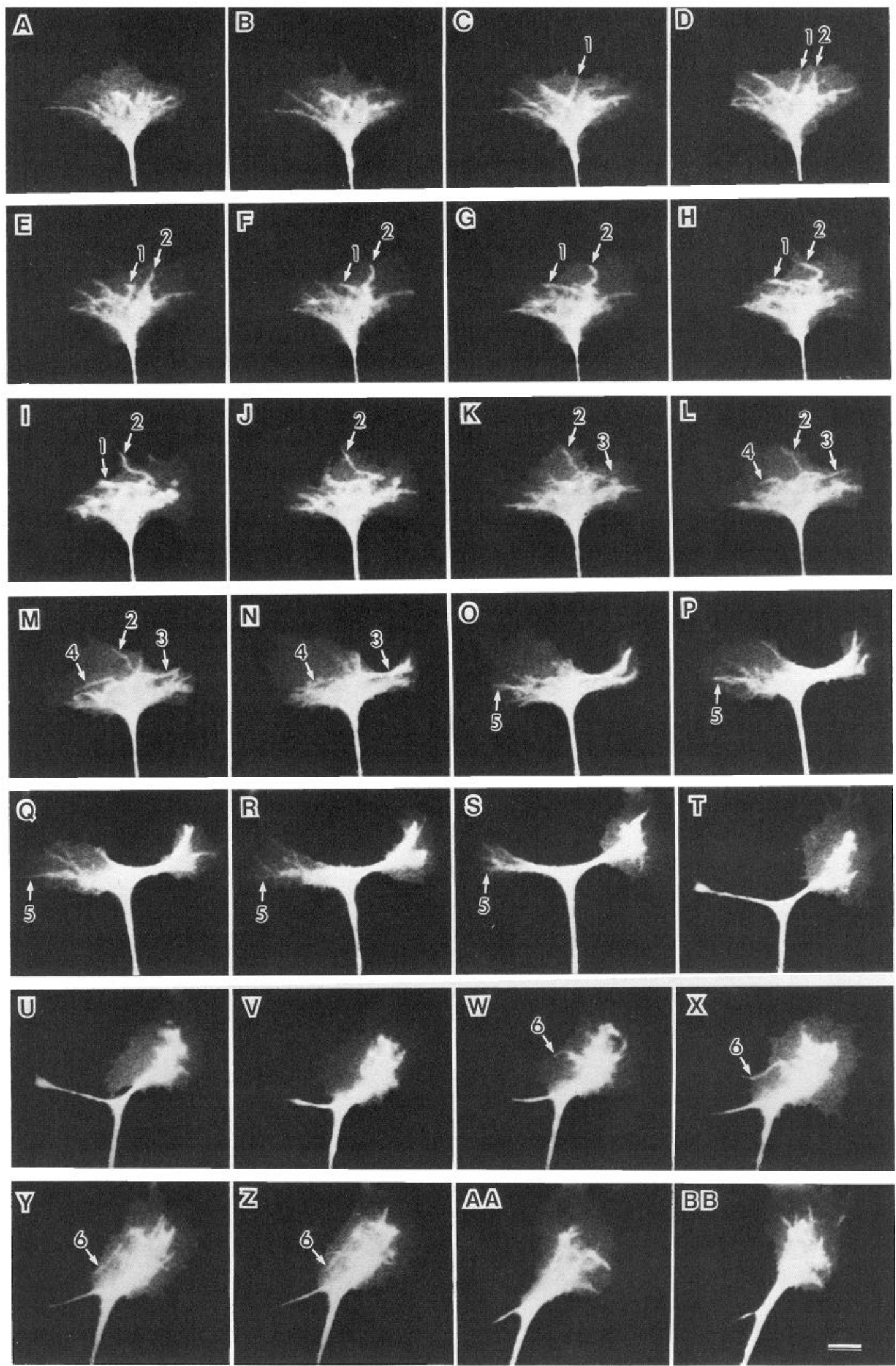

Figure 5. Time-lapse sequence of branching growth cone with $\mathrm{DiOC}_{6}(3)$-fluorescent processes demonstrating a variety of behaviors. $A-B B$ are selected images spanning $1.5 \mathrm{hr}$. Intervals were $\sim 1.5 \mathrm{~min}$ for $A-M$ and $2-8 \mathrm{~min}$ for $M-B B$. Process 1 extended to margin $(C)$, retracted $(D, E)$, re-extended with a different orientation $(E-G)$, thickened $(H, I)$, and retracted again. Process 2 extended $(D, E)$, became bent as it aligned with a small filopodium $(F-H)$, extended into the base of the filopodium $(I, J)$, and retracted $(K-N)$. Process 3 emerged as an exceedingly thin process $(K)$ that became thicker $(L, M)$ and was engulfed by the advancing fluorescent mass $(N)$. Process 4 extended laterally within the growth cone $(L$, $M)$ and was swept backward into the fluorescent mass $(N)$. Process 5 remained within the retracting branch of the growth cone $(O-S)$. Process 6 extended backward following protrusion of the rear margin. The initially thin process $(W)$ became thicker as it extended to the base of a filopodium $(X)$ and was swept centrally into the fluorescent mass $(Y, Z)$. Scale bar, $10 \mu \mathrm{m}$. 

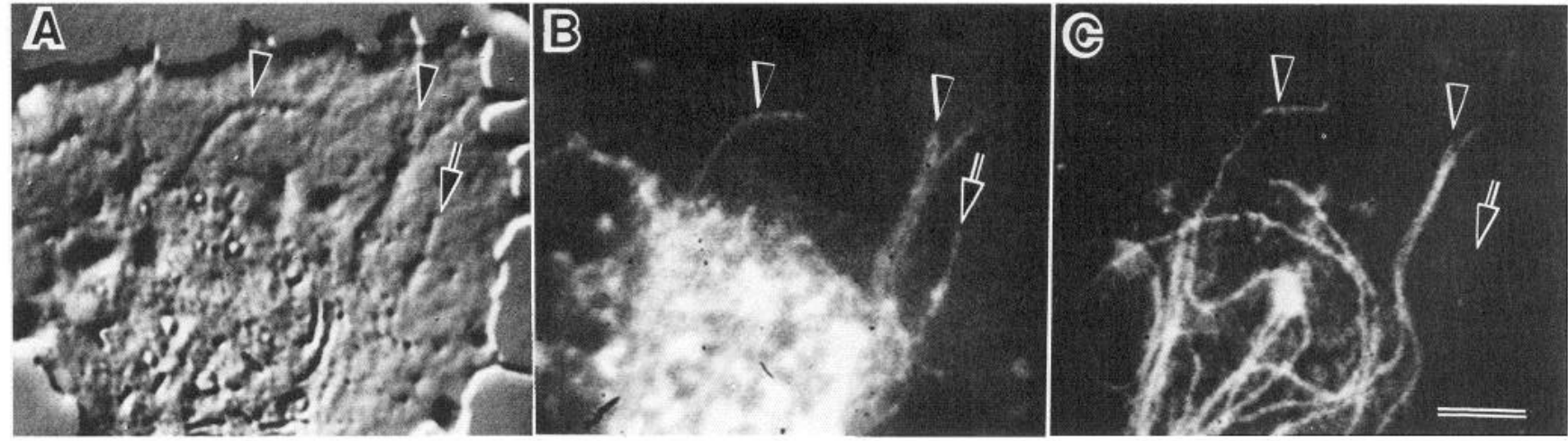

Figure 6. Correspondence of thicker-appearing central-type cytoplasmic extensions (CEs) with DiOC $_{6}(3)$ fluorescence and/or anti-tubulin immunofluorescence. $A$, Video-enhanced differential interference contrast image of fixed growth cone. Note the thickened cytoplasmic processes (arrow and arrowheads) that extend into the periphery. $B, \mathrm{DiOC}_{6}(3)$ fluorescence image. $C$, Anti-tubulin immunofluorescence image revealing microtubules (MTs). The CEs often correspond to both $\operatorname{DiOC}_{6}(3)$ and MTs (arrowheads). In another instance (arrows), anti-tubulin immunofluorescence was lacking. Scale bar, $3.6 \mu \mathrm{m}$.

Despite the lack of any correlation between extension/retraction of the thin fluorescent processes and local growth cone protrusion, advance of the growth cone as a whole leading to neurite elongation did consistently exhibit the following sequence. Processes emerged from the fluorescent mass, extended into the periphery, and became progressively thicker in appearance (Fig. 4, \#2; Fig. 5, \#1). Occasionally, some of these thickened processes subsequently retracted (Fig. 5, \#1). However, in advancing growth cones we observed processes to continue to increase in thickness and become filled with or engulfed by the fluorescent mass as it advanced forward (Fig. 5, \#3).

\section{Observations of growth cones using VEC-DIC microscopy}

Our observations that finger-like processes of $\mathrm{DiOC}_{6}(3)$-fluorescence have a dynamic distribution and are highly colocalized with microtubules prompted us to investigate the relationship of the $\mathrm{DiOC}_{6}(3)$-fluorescent processes and microtubules to organelle transport. We examined organelle movements in growth cones using VEC-DIC microscopy. We found that a mass of moving organelles was localized to the growth cone base; this region of the growth cone has previously been defined as the central cytoplasmic domain (C-domain; Bridgman et al., 1986; Forscher et al., 1987). The majority of organelles were in apparent Brownian motion, although many organelles exhibited directed transport from the distal neurite into the growth cone base (orthograde transport) or from the growth cone into the neurite (retrograde transport).

In several growth cones we were able to distinguish what appeared to be extensions of central-type cytoplasm, that is, fingerlike processes that projected into the growth cone periphery from the $\mathrm{C}$-domain and that appeared thicker than the surrounding P-domain cytoplasm. We found that even after fixation, the $\mathrm{C}$ - and $\mathrm{P}$-domains of growth cones, including the central-type cytoplasmic extensions, remained distinct when viewed with VEC-DIC microscopy. Thus, we examined the distribution of $\mathrm{DiOC}_{6}(3)$-fluorescence and immunofluorescently labeled microtubules in relation to the central-type cytoplasmic extensions within fixed growth cones. Many of the cytoplasmic extensions corresponded to both microtubules and $\mathrm{DiOC}_{6}(3)$ fluorescence, although some corresponded to only $\mathrm{DiOC}_{6}(3)$ or microtubules (Fig. 6). All of the $\mathrm{DiOC}_{6}$ (3)-fluorescent processes and corresponding central-type cytoplasmic extensions, includ- ing those not associated with microtubules, appeared continuous along their length. Bright punctate spots of fluorescence associated with $\mathrm{DiOC}_{6}(3)$-fluorescent processes often appeared as individual spherical or ovoid organelles in VEC-DIC images.

We examined the behavior of the cytoplasmic extensions in living growth cones and found a striking similarity with the behavior of $\mathrm{DiOC}_{6}(3)$-fluorescent processes: they lengthened, retracted, and changed configuration, sometimes becoming aligned with filopodia (Fig. 7, $A-H$ ). The cytoplasmic extensions advanced into the periphery as smooth, unbroken processes, and individual vesiclelike organelles were observed to translocate along some of these processes. In fact, in growth cones in which the C-domain extensions were clearly discernible, directed transport of vesicles into the periphery occurred only through the $\mathrm{C}$-domain extensions. Emergence of the cytoplasmic extensions invariably preceded directed transport of organelles into the periphery, although some of the extensions did not have vesiclelike transport associated with them. Some extensions retracted even after organelles translocated along their length (Fig. 7, $A-H)$.

When growth cones observed with VEC-DIC microscopy were fixed and $\mathrm{DiOC}_{6}(3)$-stained, it was revealed that $\mathrm{DiOC}_{6}(3)$ fluorescence corresponded precisely to the mass of moving organelles at the growth cone base, as well as to many of the thickerappearing cytoplasmic extensions (Fig. 7, $I, J$ ). Fluorescence was continuous along the length of all cytoplasmic extensions even though some of the extensions were dotted by bright spots.

\section{Discussion}

Specificity of DiOC 6 (3) staining in non-neuronal cells and neuronal growth cones

Within cultured non-neuronal cells, $\mathrm{DiOC}_{6}(3)$ stains a distinct reticular network, as well as mitochondria, vesiclelike organelles, and the plasma membrane (Fig. 1A: Johnson et al., 1981; Terasaki et al., 1984). However, organellar staining is characterized by differences in staining intensity such that the reticular network appears intermediate to the faintly stained plasma membrane and the intensely fluorescent mitochondria and other vesicle-like organelles (Terasaki et al., 1984). Compelling evidence indicates that the $\mathrm{DiOC}_{6}(3)$-stained reticular network is indeed the endoplasmic reticulum: (1) $\mathrm{DiOC}_{6}(3)$ staining is sensitive to extraction of cells with detergent but not to treatments 

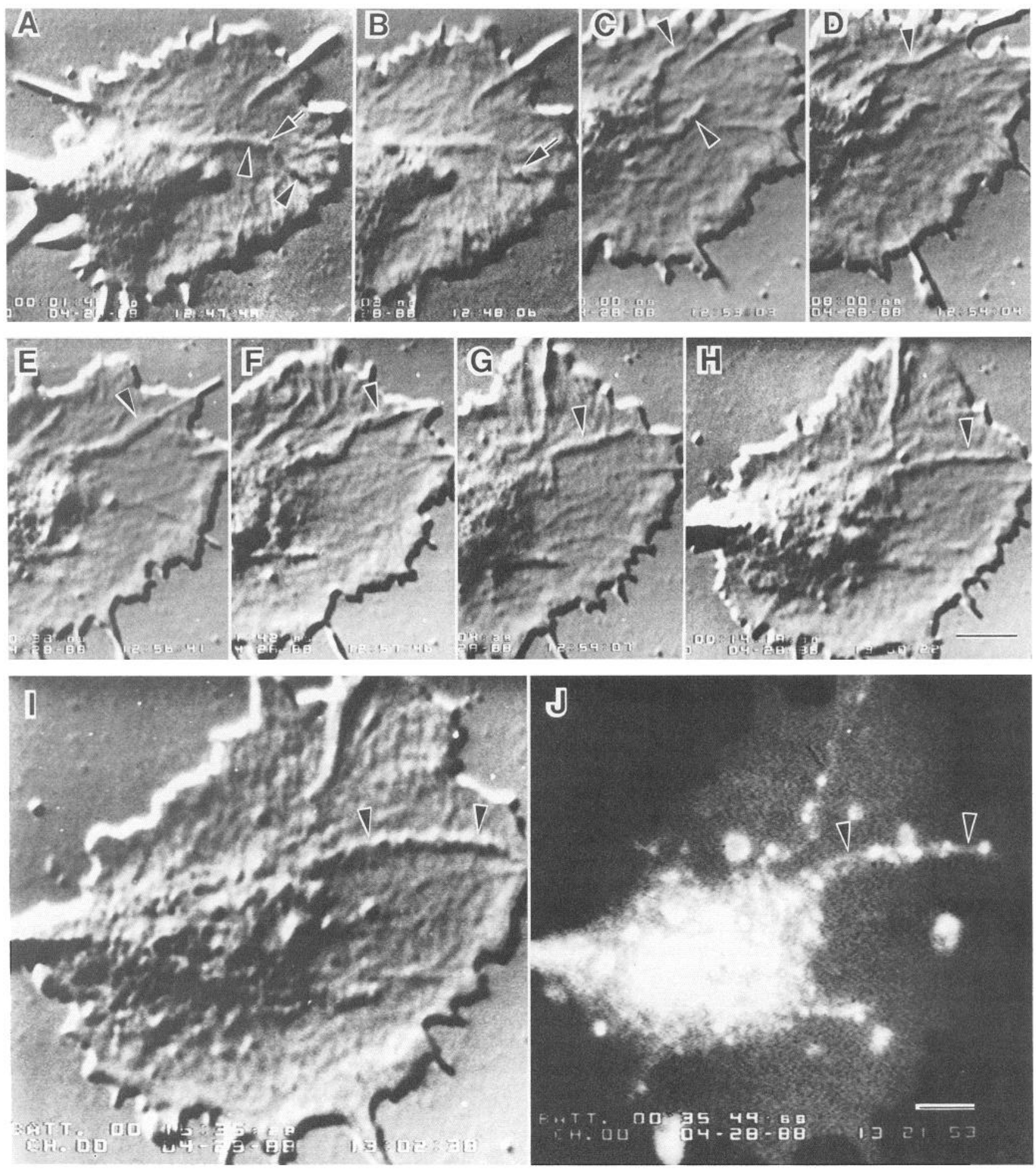

Figure 7. Sequence of video images of a single growth cone observed for $\sim 15 \mathrm{~min}$, then fixed, and stained with $\mathrm{DiOC}(3)$. $A-H$, Video sequence demonstrating movement of an organelle (arrows) and central-type cytoplasmic extensions (CEs; arrowheads) within the growth cone periphery. Organelles moved orthogradely along a $\mathrm{CE}(A, B)$, which eventually retracted. Different CEs extended and retracted concomitantly. Note the extension, alignment with a filopodium, and lateral movement of a prominent CE (upper arrowheads, $C$ - $H$ ). I, VEC-DIC image immediately after fixation. $J$, Fluorescence image demonstrating the correspondence of $\mathrm{DiOC}_{6}(3)$ with a prominent $\mathrm{CE}$ (compare $I$ and $J$, arrowheads). Fluorescence was continuous along the $\mathrm{CE}$, and bright punctations presumably represented staining of transported organelles. Larger isolated bright spots in periphery may indicate local distortion of plasmalemma after fixation and staining. Scale bars: $A-H, 3.6 \mu \mathrm{m} ; I, J, 2.5 \mu \mathrm{m}$. 
that affect protein conformation (Terasaki et al., 1984); (2) the morphology and distribution of the $\mathrm{DiOC}_{6}(3)$-fluorescent reticular pattern appears similar to networks of membranous tubules and cisternae in cells observed with whole-mount and thinsection electron microscopy (Porter et al., 1945; Terasaki et al., 1984); (3) microtubules are highly colocalized with curvilinear elements of the $\operatorname{DiOC}_{6}(3)$-stained reticular structure (Terasaki et al., 1986) and with tubules of ER observed in cells using whole-mount electron microscopy (Buckley and Porter, 1975), although the cellular distributions of microtubules and intermediate filaments as revealed by immunofluorescence are distinct from that of the $\operatorname{DiOC}_{6}(3)$-stained reticular structures (Terasaki et al., 1986); (4) early studies (Rose and Pomerat, 1960; Buckley, 1964) indicated that phase-contrast microscopy could be used with some success to visualize the ER in living cells. Recent work (Lee and Chen, 1988) using fluorescence microscopy of living cells has demonstrated that elements of the $\mathrm{DiOC}_{6}(3)$-fluorescent reticular structure exhibit movements that are similar to movements of the reticular structures observed with phase contrast (Buckley, 1964), and in single fixed cells the phase-contrast and fluorescence images of the reticular structures are exactly superimposable (Terasaki et al., 1984). Thus, $\operatorname{DiOC}_{6}(3)$ stains the ER and other intracellular membranous organelles, though with varying intensity.

The pattern of $\mathrm{DiOC}_{6}(3)$ staining within neuronal growth cones differs from that observed in thinly spread regions of cultured non-neuronal cells. Reticular networks were not observed in growth cones; instead, a fluorescent mass occupied the growth cone base and fingerlike processes projected into the periphery against a background of faint plasma membrane staining. The mass of fluorescence at the base corresponded precisely to the domain of moving particles observed in living growth cones (Fig. 6), and wholc-mount and thin-scetion clcctron microscopy have previously indicated that this cytoplasmic region (the central or C-domain) of growth cones contains a variety of membrane-bound organelles, including vesicles, mitochondria, and ER (Forscher et al., 1987; Bridgman and Dailey, 1989). Thus, the $\mathrm{DiOC}_{6}(3)$-fluorescent mass at the base of growth cones probably represents staining of a plethora of membrane-bound organelles such that clear resolution of individual organelles is often difficult or impossible.

The fluorescent fingerlike processes within the growth cone periphery are distinct from mitochondria, which have a greater fluorescence intensity, somewhat stouter morphology, and normally occupy the central domain of growth cones (Bunge, 1973; Bridgman and Dailey, 1989). As in non-neuronal cells (Terasaki et al., 1986), the distribution of $\operatorname{DiOC}_{6}(3)$ is similar to, but distinct from, microtubules (Fig. 2), and quite different from neuro- (intermediate) filaments, which do not penetrate into the periphery of growth cones (Shaw et al., 1981; M. E. Dailey and P. C. Bridgman, unpublished observations). The $\operatorname{DiOC}_{6}(3)$-fluorescent processes may represent staining of strings of vesicles or vesiclelike organelles (Letourneau, 1979). However, our observations indicate that vesiclelike organelles seen in VEC-DIC images very often appear as bright punctate spots in fluorescence images, and although punctate spots are sometimes associated with the fluorescent processes, the processes stain less intensely than vesicles and the staining is continuous rather than punctate.

The continuous, unbroken nature of the fluorescent processes and corresponding central-type cytoplasmic extensions suggest that the processes contain a continuous membranous element. This raises the possibility that a tubular or tubulovesicular com- ponent of the endoplasmic reticulum is the underlying membranous structure. In support of this, Letourneau and Ressler (1983) have observed with the electron microscope the close parallel alignment of smooth-membraned saccules with microtubules in the periphery of growth cones, and this correlates well with our finding that continuous $\mathrm{DiOC}_{6}(3)$-fluorescent processes and microtubules are highly colocalized. Thus, an attractive hypothesis is that the $\mathrm{DiOC}_{6}(3)$-fluorescent processes within the periphery of growth cones represent staining of membranous organelles which include tubular or tubulovesicular elements of the ER as well as some vesiclelike organelles. Since thin fluorescent processes were observed to progressively thicken in living cells, it may be that thickerring occurs by invasion of additional tubular or vesicular membranous organelles.

\section{Microtubules and the dynamic distribution of organelles}

Dynamic rearrangements and length excursions of the Di$\mathrm{OC}_{6}(3)$-fluorescent processes within the periphery of living growth cones presumably reflect changes in the spatial distribution of membranous organelles. The codistribution of membranous organelles $\left[\mathrm{DiOC}_{6}(3)\right.$ fluorescence] and microtubules within the periphery of growth cones is consistent with the idea that microtubules are the substrate for directed organelle transport (Hayden and Allen, 1984; Schnapp et al., 1985) in this growth cone region. Thus, extension and retraction of the $\mathrm{DiOC}_{6}(3)$ fluorescent processes may reflect translocations of membranous organelles along existing microtubule segments. This is likely for at least some of the vesicular components of the fluorescent processes since with VEC-DIC microscopy we observed vesiclelike organelle translocations along preexisting central-type cytoplasmic processes. The tubular component of processes may likewise be transported along microtubules since, in non-neuronal cells, tubular elements of ER exhibit dynamic spatial changes (Buckley, 1964; T ee and Chen, 1988), and experimental work with cell-free cultures indicates that ER-like tubules can translocate along microtubules (Dabora and Sheetz, 1988).

The length excursions and rearrangements of the $\mathrm{DiOC}_{6}(3)$ fluorescent processes may reflect microtubule dynamics (Keith et al., 1981; Sammak and Borisy, 1988a, b; Schulze and Kirschner, 1988). Growth of microtubules in fibroblast lamellae is characterized by tempered dynamic instability, and individual microtubules exhibit independent growth/shrinkage, lateral mobility, and bending (Sammak and Borisy, 1988a, b). These features confer to microtubules a pattern of behavior that is strikingly similar to the behavior of $\mathrm{DiOC}_{6}(3)$-fluorescent processes. Terasaki et al. (1986) have investigated the relationship of ER and microtubule dynamics in cultured cells and found that microtubule polymerization and ER-tubule extension occur together at the level of resolution of the fluorescence microscope. Furthermore, reticular networks of ER retract back slowly after microtubule depolymerization (Terasaki et al., 1986), and it is possible that free-ending tubules may reflect more closely the dynamics of associated microtubules. The slight disparity of $\mathrm{DiOC}_{6}(3)$-fluorescence and microtubule distribution we observed in growth cones (Fig. 2) may have arisen from a time lag in the response (extension/retraction) of the membranous organelles (ER) to rapid microtubule polymerization/depolymerization. Real-time imaging of both $\mathrm{DiOC}_{6}(3)$ fluorescence and microtubules within single growth cones should help determine more precisely the spatiotemporal relationship of membranous organelles and microtubules. 


\section{Distribution of organelles in relation to growth cone function}

Previous observations of living growth cones using high-resolution video microscopy indicated that neurite elongation involves progressive advance of vesiclelike organelles associated with the central-type cytoplasm (C-domain) into organelle-poor regions (P-domain) of the growth cone (Goldberg and Burmeister, 1986; Aletta and Greene, 1988). Organelles were observed to invade the P-domain by long excursions, short steps, or Brownian motion, although it was noted that some "factor, or factors" seemed to limit the ingress of vesiclelike organelles (Goldberg and Burmeister, 1986). Forscher et al. (1987) studied growth cones of Aplysia bag cell neurons and found that, under normal culture conditions, "individual organelles or groups of several organelles linked together" occasionally moved into the $\mathrm{P}$-domain, sometimes reversing direction and returning to the C-domain.

We find that organelle transport pathways within the growth cone periphery invariably corresponded with $\mathrm{DiOC}_{6}(3)$ fluorescence. Even when only a few vesicles translocated along a particular pathway, the $\mathrm{DiOC}_{6}(3)$ fluorescence was continuous along the pathway, suggesting the presence of an ER-like tubular membranous element. In addition, some of the central-type cytoplasmic extensions within the growth cone periphery lacked obvious organelle movements but, nevertheless, were colocalized with processes of $\mathrm{DiOC}_{6}(3)$ fluorescence. One idea consistent with these findings is that advance of ER-like organelles may precede advance of vesiclelike organelles into the P-domain. If so, advance of the ER-like organelles in association with microtubules may contribute to the establishment and/or maintenance of transport lanes for the advance of other organelles. Perhaps the ER-like organelles regulate local calcium levels and thereby alter the polymerization or stability of microtubules (Schliwa et al., 1981; Keith et al., 1983) or the organization of the actin-containing cytoskeletal meshwork (Taylor and Condeelis, 1979; Yin and Stossel, 1979; Taylor et al., 1980). Interestingly, it was recently proposed that the diverse activities in which growth cones are involved (e.g., motility and elongation) may be differentially regulated by local calcium concentrations within defined growth cone regions (Kater et al., 1988). It may be that spatially dynamic calcium-sequestering and -releasing organelles contribute to the precise spatiotemporal regulation of intracellular calcium required for coordinate activity.

In summary, our observations indicate that organelles advance within growth cones along distinct pathways that are spatially dynamic, sometimes transient, and highly colocalized with microtubules. Furthermore, tubular membranous organelles translocate along pathways into the growth cone periphery before vesiclelike organelles. These observations suggest a sequence of events for advance of membranous organelles in growth cones that may contribute to directed axon elongation: (1) microtubules extend randomly into the growth cone periphery; (2) ER tubules coextend with, or translocate along, the microtubules; (3) microtubules with associated ER tubules may move laterally into a more preferential orientation (i.e., alignment with filopodia or lamellipodia); (4) other (vesiclelike) membranous organelles then translocate into the periphery along microtubules. Organelles would continue to advance if the microtubule remained extended; however, microtubule depolymerization would lead to retraction of organelles that had previously advanced. Eventually, the continuous transport of membranous organelles along microtubules that remain extended results in merging of the fingers with the fluorescent mass, thereby effectually advancing the mass forward. This possible sequence leaves unanswered the important question: What is the signal, or signals, that determines whether a microtubule will remain extended long enough for this maturation process to occur? Because of the close association of the ER with microtubules, it seems reasonable to propose that local control of calcium levels by the ER plays at least a partial role in microtubule stabilization. However, exactly how this might occur is not straightforward since it would require the function of the ER to be under very strict spatial control. Obviously more work is needed to fully understand how directed growth occurs and what role microtubules and organelles such as the endoplasmic reticulum play in this process.

\section{References}

Aletta, J. M., and L. A. Greene (1988) Growth cone configuration and advance: A time-lapse study using video-enhanced differential interference contrast microscopy. J. Neurosci. 8: 1424-1435.

Bridgman, P. C., and M. E. Dailey (1989) Organization of myosin and actin in rapid frozen nerve growth cones. J. Cell Biol. 108: 95109.

Bridgman, P. C., B. Kachar, and T. S. Reese (1986) The structure of cytoplasm in directly frozen cultured cells. II. Cytoplasmic domains associated with organelle movements. J. Cell Biol. 102: 1510-1521.

Buckley, I. K. (1964) Phase contrast observations on the endoplasmic reticulum of living cells in culture. Protoplasma 59: 569-588.

Buckley, I. K., and K. R. Porter (1975) Electron microscopy of critical point dried whole cultured cells. J. Microsc. 104: 107-120.

Bunge, M. B. (1973) Fine structure of nerve fibers and growth cones of isolated sympathetic neurons in culture. J. Cell Biol. 56: 713-735.

Burton, P. R., and L. A. Laveri (1985) The distribution, relationships to other organelles, and calcium-sequestering ability of smooth endoplasmic reticulum in frog olfactory axons. J. Neurosci. 5: 30473060.

Carafoli, E. (1987) Intracellular calcium homeostasis. Annu. Rev. Biochem. 56: 395-433.

Cohan, C. S., and S. B. Kater (1986) Suppression of neurite elongation and growth cone motility by electrical activity. Science 232: 1638 1640 .

Cohan, C. S., J. A. Connor, and S. B. Kater (1987) Electrically and chemically mediated increases in intracellular calcium in neuronal growth cones. J. Neurosci. 7: 3588-3599.

Connor, J. A. (1986) Digital imaging of free calcium changes and of spatial gradients in growing processes in single, mammalian central nervous system cells. Proc. Natl. Acad. Sci. USA 83: 6179-6183.

Dabora, S. L., and M. P. Sheetz (1988) The microtubule-dependent formation of a tubulovesicular network with characteristics of the ER from cultured cell extracts. Cell 54: 27-35.

Dailey, M. E., and P. C. Bridgman (1987) Fluorescence imaging of endoplasmic reticulum in growth cones of cultured rat superior cervical ganglion neurons. Soc. Neurosci. Abstr. 13: 1477.

Forscher, P., L. K. Kaczmarek, J. Buchanan, and S. J. Smith (1987) Cyclic AMP induces changes in distribution and transport of organelles within growth cones of Aplysia bag cell neurons. J. Neurosci. 7: 3600-3611.

Goldberg, D. J., and D. W. Burmeister (1986) Stages in axon formation: Observations of growth of Aplysia axons in culture using video-enhanced contrast-differential interference contrast microscopy. J. Cell Biol. 103: 1921-1931.

Gundersen, R. W., and J. N. Barrett (1980) Characterization of the turning response of dorsal root neurites toward nerve growth factor. J. Cell Biol. 87: 546-554.

Hayden, J. H., and R. D. Allen (1984) Detection of single microtubules in living cells: Particle transport can occur in both directions along the same microtubule. J. Cell Biol. 99: 1785-1793.

Henkart, M. P., T. S. Reese, and F. J. Brinley, Jr. (1978) Endoplasmic reticulum sequesters calcium in the Squid giant axon. Science 202: 1300-1303.

Johnson, L. V., M. L. Walsh, B. J. Bockus, and L. B. Chen (1981) 
Monitoring of relative mitochondrial membrane potential in living cells by fluorescence microscopy. J. Cell Biol. 88: 526-535.

Kater, S. B., M. P. Mattson, C. Cohan, and J. Connor (1988) Calcium regulation of the neuronal growth cone. TINS 11: 315-321.

Keith, C., J. Feramisco, and M. Shelanski (1981) Direct visualization of fluorescein-labeled microtubules in vitro and in microinjected fibroblasts. J. Cell Biol. 88: 234-240.

Keith, C., M. Paola, F. R. Maxfield, and M. L. Shelanski (1983) Microinjection of $\mathrm{Ca}^{++}$-calmodulin causes a localized depolymerization of microtubules. J. Cell Biol. 97: 1918-1924.

Lee, C., and L. B. Chen (1988) Dynamic behavior of endoplasmic reticulum in living cells. Cell $54: 37-46$.

Letourneau, P. C. (1979) Cell-substratum adhesion of neurite growth cones and its role in neurite elongation. Exp. Cell Res. 124: 127-138.

Letourneau, P. C., and A. H. Ressler (1983) Differences in the organization of actin in growth cones compared with the neurites of cultured neurons from chick embryos. J. Cell Biol. 97: 963-973.

Lipscombe, D., D. V. Madison, M. Poenie, H. Reuter, R. Y. Tsien, and R. W. Tsien (1988a) Spatial distribution of calcium channels and cytosolic calcium transients in growth cones and cell bodies of sympathetic neurons. Proc. Natl. Acad. Sci. USA 85: 2398-2402.

Lipscombe, D., D. V. Madison, M. Poenie, H. Reuter, R. W. Tsien, and $\mathrm{R}$. Y. Tsien (1988b) Imaging of cytosolic $\mathrm{Ca}^{2+}$ transients arising from $\mathrm{Ca}^{2+}$ stores and $\mathrm{Ca}^{2+}$ channels in sympathetic neurons. Neuron 1: $355-365$.

McBeath, E., and K. Fujiwara (1984) Microtubule kinetics in cells studied by immunofluorescence. In International Cell Biology 1984, S. Seno and Y. Okada, eds., Abstr. 4210, Academic, San Diego, CA.

Neering, I. R., and R. N. McBurncy (1984) Rolc for microsomal Ca storage in mammalian neurons? Nature 309: 158-160.

Payne, R., and A. Fein (1987) Inositol 1,4,5 trisphosphate releases calcium from specialized sites within Limulus photoreceptors. J. Cell Biol. 104: 933-937.

Porter, K. R., A. Claude, and E. F. Fullam (1945) A study of tissue culture cells by electron microscopy. J. Exp. Med. 81: 233-241.

Rees, R. P., and T. S. Reese (1981) New structural features of freezesubstituted neuritic growth cones. Neuroscience 6: 247-254.

Rose, G. G., and C. M. Pomerat (1960) Phase contrast observations of the endoplasmic reticulum in living tissue cultures. J. Biophys. Biochem. Cytol. 8: 423-430.

Sammak, P. J., and G. G. Borisy (1988a) Direct observation of microtubule dynamics in living cells. Nature 332: 724-726.
Sammak, P. J., and G. G. Borisy (1988b) Detection of single fluorescent microtubules and methods for determining their dynamics in living cells. Cell Mot. Cytoskel. 10: 237-245.

Schliwa, M., U. Euteneuer, J. C. Bulinski, and J. G. Izant (1981) Calcium lability of cytoplasmic microtubules and its modulation by microtubule-associated proteins. Proc. Natl. Acad. Sci. USA 78: 10371041 .

Schnapp, B. J., R. D. Vale, M. P. Sheetz, and T. S. Reese (1985) Single microtubules from squid axoplasm support bidirectional movement of organelles. Cell 40:455-462.

Schulze, E., and M. Kirschner (1988) New features of microtubule behavior observed in vivo. Nature 334: 356-359.

Shaw, G., M. Osborn, and K. Weber (1981) Arrangement of neurofilaments, microtubules and microfilament-associated proteins in culturcd dorsal root ganglia cells. Eur. J. Cell Biol. 24: 20-27.

Somlyo, A. P. (1984) Cellular site of calcium regulation. Nature 309. 516-517.

Taylor, D. L., and J. S. Condeelis (1979) Cytoplasmic structure and contractility in Amoeboid cells. In International Review of Cytology, Vol. 56, G. H. Bourne and J. F. Danielli, eds., pp. 57-144, Academic, New York.

Taylor, D. L., J. R. Blinks, and G. Reynolds (1980) Contractile basis of Ameboid movement. VIII. Aequorin luminescence during Ameboid movement, endocytosis, and capping. J. Cell Biol. 86: 599-607.

Terasaki, M., J. Song, J. R. Wong, M. J. Weiss, and L. B. Chen (1984) Localization of endoplasmic reticulum in living and glutaraldehydefixed cells with fluorescent dyes. Cell 38: 101-108.

Terasaki, M., L. B. Chen, and K. Fujiwara (1986) Microtubules and the cndoplasmic reticulum are highly interdependent structures. J. Cell Biol. 103: 1557-1568.

Unger, F., I. Piscopo, J. Letizia, and E. Holtzman (1984) Uptake of calcium by the endoplasmic reticulum of the frog photoreceptor. $J$. Cell Biol. 98: 1645-1655.

Walz, B. (1982) $\mathrm{Ca}^{2+}$-sequestering smooth endoplasmic reticulum in an invertebrate photoreceptor. I. Intracellular topography as revealed by $\mathrm{OsFeCN}$ staining and in situ $\mathrm{Ca}$ accumulation. J. Cell Biol. 93. 839-848.

Yin, H. L., and T. P. Stossel (1979) Control of cytoplasmic actin gelsol transformation by gelsolin, a calcium-dependent regulatory protein. Nature 281: 583-585. 